\title{
Motor Imagery and Motor Coordination in Autism Spectrum Disorders: Similarities and Differences
}

\author{
Emanuela Nobile ${ }^{1}$, Alessandro Piedimonte ${ }^{1,2 *}$ and Roberto Keller ${ }^{3}$ \\ ${ }^{1}$ Fondazione Carlo Molo Onlus, Turin, Italy \\ ${ }^{2}$ Department of Neuroscience, University of Turin, Italy \\ ${ }^{3}$ Centre for Autism Spectrum Disorder in Adulthood ASL To2, Turin, Italy
}

\begin{abstract}
Autism spectrum disorders (ASD) are composed by a variety of developmental deficits, such as problems in verbal communication and repetitive behaviours. Together with these social deficits, the recent literature has reported difficulties in motor coordination during the everyday life of children and adolescents with ASD. These motor deficits could be linked to a possible alteration of motor simulation processes in ASD but just a few studies attempted to directly compare motor imagery tasks and actual motor coordination within these disorders and specifically using the same task. In a recent study, the relationship between explicit motor imagery and motor execution has been investigated within the same experimental paradigm. The authors employed a spatial bimanual task called "circles-lines" task where participants are asked to draw with one hand (i.e., baseline), either hands (i.e., bimanual task) or try to imagine the movements with one hand while simultaneously draw with the other (i.e., imagery task). This task is a prototype of a complex bimanual skill and shows how these two processes can be studied together within the same paradigm. In particular, results of the study highlighted how motor imagery and motor coordination only partially follow the same path of development in ASD. Indeed, ASD participants showed much lower results in the motor imagery task in comparison to the performance in actual bimanual task, which was similar to the typically developed controls. In conclusion, the data show that motor imagery and actual coordination, even though interrelated processes which share similar brain areas, can be dissociated in ASD where development of spatial coordination consolidates earlier than motor imagery.
\end{abstract}

Keywords: Autism spectrum disorders (ASD); Motor imagery; Motor coordination

\section{Introduction}

Autism spectrum disorders (ASD) represents a cohort of developmental disorders composed by a wide range of deficits, generally focused around social-communicative difficulties as well as repetitive stereotyped behaviours. However, in the last decade it has also been shown that motor impairments are of clinical and functional importance when assessing ASD. Indeed, even though motor disturbances are not considered core features of autism they still have a significant effect on people with ASD trough their everyday life. Sensory and motor impairments can be observed in infancy and thus can be considered among the first detectable behavioural problems in ASD and tend to become apparent during childhood and adolescence. A number of different motor deficits have been observed in ASD as clumsiness, postural instability, motor coordination and more in general a widespread poorer performance on motor skills tests in comparison to people without ASD [1]. These problems seem to be in part negatively correlated to language acquisition so that, for instance, adolescents with ASD who do not present any speech delay show poorer bimanual coordination in comparison with those with speech delay [2].

\section{Motor Imagery in ASD}

Interestingly, recent studies linked these motor deficits to a possible alteration of motor simulation processes in ASD [3]. The theory behind motor simulation states that all sensorimotor information related to the execution of a movement is also used by other processes such as imitation, understanding of movements performed by other people and the skill to imagine one's own movements [4]. These imagery processes has been assessed in ASD using mental transformation tasks where participants had to imagine and mentally rotate the whole body or body parts such as the hands [5]. The idea behind these tasks is that participants engaged in the mental rotation of body parts, instead of simple objects rotations, have to solve the task by mentally imagining their own movements and thus relying to what has been called "motor imagery". Results from these studies revealed that, when required to decide if a hand image in different orientations represents a left or a right hand, adolescents with ASD seem to be incapable to activate correct sensorimotor information to simulate their own actions and thus they have a worse performance in comparison to typical adolescents who correctly imagine movements of their hands [5]. It is worth to be noted that this judgment task has been classified as an "implicit" motor imagery task since no instruction is given to participants on how to judge the laterality of the hand pictures whereas other classical motor imagery tasks requires participants to imagine a specific movement focusing on its motoric aspects [6]. Implicit and explicit motor imagery seem also to involve different brain areas so that an implicit task relies on the parietal cortex as it seem to not require the involvement of motor preparation while explicit tasks are more related to the activation of the supplementary motor cortex [7].

However, just a few studies attempted to directly compare motor imagery tasks and actual motor coordination in ASD and, furthermore, these studies focused only on the implicit aspect of motor imagery involving mental rotation tasks [8]. Indeed, even though explicit motor imagery and actual motor execution share common brain areas such as the supplementary motor cortex, it has been shown in normally developed participants that the acquisition of each of these skills do not follow the same trend, since effective motor imagery skills seems to be acquired later around 10 years or even in the middle adolescence [6].

*Corresponding author: Alessandro Piedimonte, Department of Neuroscience, University of Turin, Italy, Tel: +393487647353; E-mail: ale.piedimonte@gmail.com

Received March 29, 2018; Accepted April 16, 2018; Published April 23, 2018

Citation: Nobile E, Piedimonte A, Keller R (2018) Motor Imagery and Motor Coordination in Autism Spectrum Disorders: Similarities and Differences. Int J Neurorehabilitation 5: 310. doi: 10.4172/2376-0281.1000310

Copyright: @ 2018 Nobile E, et al. This is an open-access article distributed under the terms of the Creative Commons Attribution License, which permits unrestricted use, distribution, and reproduction in any medium, provided the original author and source are credited. 


\section{Comparing Motor Execution and Motor Imagery in ASD}

A recent study aimed at bridging the gap between explicit motor imagery and motor execution within the same experimental paradigm [9]. In the present study, the authors employed a spatial bimanual task, called "circles-lines" task, which is a prototype of a complex motor skill. Within this task is possible to concurrently assess motor coordination and explicit motor imagery in ASD, asking participants to perform unimanual or bimanual movements in three conditions: continuously draw lines with their right hand (unimanual condition); draw lines with the right hand and simultaneously draw circles with the left hand (bimanual condition); draw lines with their right hand while imagining to draw circles with their left hand (imagery condition).

In particular, the advantage of the circles-lines task lies in the fact that, when participants simultaneously draw lines with the right hand and circles with the left hand, both trajectories tend to become ovals (i.e., bimanual coupling effect) and this effect can be captured by a tablet trough a distortion index called also "ovalization index" (OI). Within this paradigm is also possible to evaluate motor imagery and its effect on actual movements, asking participants to imagine movements with one hand while actually drawing with the other and thus measuring the OI derived by such mental representation of a movement. Furthermore, the paradigm allows for a direct comparison of the OI collected in the imagery condition with the OI recorded in the actual bimanual performance. Finally, another objective of the study was to evaluate motor coordination and motor imagery across both adolescents as well as adults with ASD that is to find differences in the development of the two skills.

The study showed that, when participants were asked to actually draw circles and lines simultaneously with both hands, ASD participants showed a significantly higher OI (i.e., distortion) in the bimanual condition in respect to the typical controls. However, no difference was found between ASC and controls' bimanual coupling effects, that is comparing the ratio between bimanual and unimanual distortions: a measure that can be considered an index of coordination. This first result shows that ASD participants show an almost normal coordination performance and it is in contrast with the literature where they show reduced rhythmic bimanual coordination, fine motor control as well as bimanual gesture reproduction [1]. It is possible to explain this discrepancy with the previous literature because the circles-lines task can be viewed as a gross motor task and thus it mainly involves proximal muscles (i.e. shoulder and arm movements) that are less demanding with respect to other coordination tasks involving distal muscles and production of precision grips (i.e., finger and hand movements).

On the other hand, when participants were asked to imagine to draw circles with their left hand while simultaneously drawing lines with their right hand, only typically developing adolescents and adults showed a significant coupling effect, that is a significant OI increment in the imagery condition in comparison to the unimanual condition, and this effect was significantly stronger in the typical adults in comparison to all the other groups. This second result shows that motor imagery in the ASD group is not strong enough, even during adulthood, to produce an effective distortion of the lines drawn with the right hand, while in the typical controls it is already strong to produce coupling effects during adolescence and this skill continues to improve greatly during adulthood.

\section{Conclusion}

The study presented in this mini-review confirms how it is possible to investigate within the same paradigm the development of both explicit motor imagery as well as motor execution but also demonstrates how these two processes only partially follow the same path. In particular, ASD participants showed much lower results in the motor imagery task in comparison to the performance in the actual bimanual task, where they showed similar performances in respect to the typically developed controls. As mentioned in the previous paragraph, motor imagery, investigated within the same experimental paradigm, is powerful enough to elicit an observable coupling effect (an increase of the OI) already at around age 10 and has the highest effects between 20 and 30 years old [7]. This path of development is also confirmed by previous studies with different paradigms where it was shown that motor imagery tend to be correctly used only after age 7 , since younger children tend to support motor imagery processes by performing actual movements [10]. Thus, this finding highlights that motor imagery is still immature in ASD participants and could be correlated to a more general deficit in action representation that is the ability to simulate an action or interaction with the environment. In turn, this deficit could be the basis for atypical motor behaviours in ASD [6] leading to an increased deficit in temporal as well as spatial aspects of execution [2], reflected in the paper of Piedimonte and collaborators by the increased distortion showed in the ASD group in the actual bimanual condition. Finally, this difficulty in representing specific actions could be generalized in ASD and thus lead to a reduced ability to understand and respond to complex social situations.

In conclusion, motor imagery and actual motor coordination are interrelated processes that, however, seem to be dissociated in ASD where development of spatial coordination consolidates earlier in respect to motor imagery. Future studies should try to incorporate both explicit and implicit form of motor imagery but also different tasks of motor coordination (i.e., temporal and spatial, unimanual and bimanual) to have a complete view on these two fundamental mechanisms in ASD.

\section{References}

1. Gowen E, Hamilton A (2013) Motor abilities in autism: A review using a computational context. J Autism Dev Disord 43: 323-344.

2. Barbeau EB, Meilleur AAS, Zeffiro TA, Mottron L (2015) Comparing motor skills in autism spectrum individuals with and without speech delay. Autism Res 8: 682-693.

3. Gallese V, Rochat MJ, Berchio C (2013) The mirror mechanism and its potential role in autism spectrum disorder. Dev Med Child Neurol 55: 15-22.

4. Decety J, Grèzes J (2006) The power of simulation: Imagining one's own and other's behavior. Brain Res 1079: 4-14.

5. Conson M, Mazzarella E, Frolli A, Esposito D, Marino N, et al (2013) Motor imagery in Asperger syndrome: Testing action simulation by the hand laterality task. PLoS One 8: e70734.

6. Piedimonte A, Garbarini F, Rabuffetti M, Pia L, Berti A (2014) Executed and imagined bimanual movements: A study across different ages. Dev Psychol 50: 1073-1080

7. Hétu S, Grégoire M, Saimpont A, Coll M-P, Eugène F, et al (2013) The neura network of motor imagery: An ALE meta-analysis. Neurosci Biobehav Rev 37: 930-949.

8. Hirata S, Okuzumi H, Kitajima Y, Hosobuchi T, Nakai A, et al (2015) Relationship between motor skill impairments and motor imagery ability in children with autism spectrum disorders: A pilot study using the hand rotation task. Psychology 6: 752-759.

9. Piedimonte A, Conson M, Frolli A, Bari S, Della Gatta F, et al (2018) Dissociation between executed and imagined bimanual movements in autism spectrum conditions. Autism Res 11: 376-384.

10. Molina M, Tijus C, Jouen F (2008) The emergence of motor imagery in children. J Exp Child Psychol 99: 196-209. 